\title{
New general integral transform via Atangana-Baleanu derivatives
}

\author{
M. Meddahi ${ }^{1}$, H. Jafari ${ }^{2,3,4^{*}}$ (iD and M.N. Ncube ${ }^{3}$
}

${ }^{\text {"Correspondence: }}$

jafari.usern@gmail.com

1 Department of Mathematics, University of Hassiba Benbouali,

Chlef, Algeria

${ }^{2}$ Department of Mathematics, University of Mazandaran, Babolsar, Iran

Full list of author information is available at the end of the article

\begin{abstract}
The current paper is about the investigation of a new integral transform introduced recently by Jafari. Specifically, we explore the applicability of this integral transform on Atangana-Baleanu derivative and the associated fractional integral. It is shown that by applying specific conditions on this integral transform, other integral transforms are deduced. We provide examples to reinforce the applicability of this new integral transform.
\end{abstract}

MSC: $34 \mathrm{A0} 08 ; 65 \mathrm{M} 70 ; 11 \mathrm{~B} 68$

Keywords: Fractional derivative; Laplace transform; Mittag-Lefler function; Atangana-Baleanu derivative; Fractional differential equation

\section{Introduction}

Fractional calculus is regarded as an extension of integer calculus in the sense that it permits the order of the derivative or integral to be a fraction. The idea of having a fractional order derivative did not make practical sense in the real world, thus the subject of fractional calculus was mainly reserved for mathematicians for a long time since its discovery [1-3].

However, researchers have come to realise that models constructed from fractional calculus can successfully represent real world problems and sometimes yield better results compared to models from the integer calculus. Some useful results from fractional calculus models appear in engineering, physics, biology and economics [1-14].

The definition of the fractional derivative is in itself a developing concept. Numerous definitions have been suggested for the fractional derivative, with almost each definition possessing some form of deficiency. It is generally believed that the choice of the derivative used is dictated by the situation that is being modelled.

Since it was discovered that the fractional derivative can be successfully applied to practical problems, it is the Caputo derivative that has been used the most. The only shortcoming of the Caputo derivative is the singularity issue. Other fractional derivatives that have this singularity problem are Riemann-Liouville, Caputo-Hadamard, and Riesz (see $[1-3,15,16])$. In a bid to address the singularity concern, the Caputo-Fabrizio derivative was proposed $[17,18]$, this derivative eliminated the singularity problem through the use of an exponential kernel. Atangana and Baleanu replaced the exponential kernel by

(c) The Author(s) 2021. This article is licensed under a Creative Commons Attribution 4.0 International License, which permits use sharing, adaptation, distribution and reproduction in any medium or format, as long as you give appropriate credit to the original author(s) and the source, provide a link to the Creative Commons licence, and indicate if changes were made. The images or other third party material in this article are included in the article's Creative Commons licence, unless indicated otherwise in a credit line to the material. If material is not included in the article's Creative Commons licence and your intended use is not permitted by statutory regulation or exceeds the permitted use, you will need to obtain permission directly from the copyright holder. To view a copy of this licence, visit http://creativecommons.org/licenses/by/4.0/. 
the Mittag-Leffler function to create another non-singular kernel derivative called the Atangana-Baleanu derivative. More detailed discussions encompassing both theory and applications of the non-singular derivatives are found in [19-24]. In [5, 25-28], the authors discuss the uniqueness and existence of fractional differential equations.

To fully harness the capability of fractional differential equations in modelling problems that arise in the real world, it is imperative that we have methods of solutions that are computationally inexpensive, easily accessible and highly accurate. Integral transforms are some of the techniques that have proven their worth, as they are regarded to be easy to implement and demand reasonable labour in terms of computations.

Integral transforms offer an alternative to integration in the solution of differential equations. The integral transform maps the domain of the original problem into a different domain consisting of an algebraic equation that is normally easy to manipulate. Taking the inverse of the new domain results in the solution of the original problem [29].

There are different types of integral transforms that are used in the solution of differential equations, but it is the Laplace transform that is mostly applied. Most of the integral transforms that have been suggested are extensions of the Laplace transform. Some of the integral transforms that are closely related to the Laplace transform are the Elzaki transform, Sumudu transform, Shehu transform, etc. [30-34].

Recently, a more generalized integral transform has been introduced by the second author [35]. Imposing specific conditions on this integral transform yields other integral transforms, for example, the Laplace transform, natural transform, Elzaki transform and Sawi transform [35]. To get a deeper insight into the properties and applications of this new general integral transform, we refer the reader to [35].

Our main intention in this research is to investigate the application of this new generalized integral transform in the solution of differential equations involving the AtanganaBaleanu derivative.

We structure the rest of our work in the following manner. In the next section, we provide some important mathematical concepts that will form the basis for our research. We then follow by presenting the main results of the research in section three. To reinforce the theoretical aspects of our work, we provide applications in the fourth section. A concise summary of our research findings is provided in the last section.

\section{Preliminaries}

Throughout, set

$$
A=\left\{f(t): \exists M>0, k>0,|f(t)| \leq M e^{k t}, \text { if } t \geq 0\right\},
$$

and suppose that $f(t)$ is an integrable function defined on the set $A$.

Definition 1 ([35]) Consider the functions $\varphi(s), \psi(s): \mathbb{R}^{+} \longrightarrow \mathbb{R}^{+}$such that $\varphi(s) \neq 0$ for all $s \in \mathbb{R}^{+}$. The new general integral transform of the function $f(t)$ denoted by $\mathcal{F}_{\mathrm{J}}(s)$ is defined by

$$
T\{f(t), s\}=\mathcal{F}_{\mathrm{J}}(s)=\varphi(s) \int_{0}^{\infty} f(t) e^{-\psi(s) t} d t
$$


with the corresponding inverse,

$$
\mathcal{F}_{\mathrm{J}}^{-1}(s)=T^{-1}\left\{\varphi(s) \int_{0}^{\infty} f(t) e^{-\psi(s) t} d t\right\}=f(t) .
$$

The integral transform (1) exists for all $\psi(s)>k$. It is simple to check that the new general integral transform is a linear operator and has many properties that are similar to other integral transforms, more detailed discussion on this can be found in [35].

Theorem 1 The integral transform $\mathcal{F}_{\mathrm{J}}(s)$ of the derivative of $f(t)$ is given as [35],

$$
T\left\{f^{(n)}(t), s\right\}=\psi^{n}(s) \mathcal{F}_{\mathrm{J}}(s)-\varphi(s) \sum_{k=0}^{n-1} \psi^{n-1-k}(s) f^{(k)}(0), \quad \varphi(s), \psi(s)>0 \forall n \in \mathbb{N} .
$$

Theorem 2 If $\mathcal{F}_{\mathrm{J}}(s)$ and $\mathcal{H}_{\mathrm{J}}(s)$ are general integral transforms of $f(t)$ and $h(t)$, respectively, then

$$
T\{f * h\}=\frac{1}{\varphi(s)} \mathcal{F}_{\mathrm{J}}(s) \cdot \mathcal{H}_{\mathrm{J}}(s) .
$$

Moreover,

$$
T^{-1}\{f \cdot h\}=\varphi(s) T^{-1}\{f\} * T^{-1}\{h\} .
$$

Proof We have

$$
f * h=\int_{0}^{\infty} f(\tau) h(t-\tau) d \tau .
$$

Using the new general transform and the Leibniz theorem, we obtain

$$
\begin{aligned}
T\{f * h\} & =T\left\{\int_{0}^{\infty} f(\tau) h(t-\tau) d \tau\right\}=\varphi(s) \int_{0}^{\infty}\left[\int_{0}^{\infty} f(\tau) h(t-\tau) d \tau\right] e^{-\psi(s) t} d t \\
& =\varphi(s) \int_{0}^{\infty} f(\tau)\left[\int_{0}^{\infty} h(t-\tau) e^{-\psi(s) t} d t\right] d \tau,
\end{aligned}
$$

by setting $u=t-\tau$, we get

$$
\begin{aligned}
T\{f * h\} & =\varphi(s) \int_{0}^{\infty} f(\tau) e^{-\psi(s) \tau}\left[\int_{0}^{\infty} h(u) e^{-\psi(s) u} d t\right] d \tau \\
& =\varphi(s) \int_{0}^{\infty} f(\tau) e^{-\psi(s) \tau} d \tau \times \frac{1}{\varphi(s)} \mathcal{H}_{\mathrm{J}}(s) \\
& =\frac{1}{\varphi(s)} \mathcal{F}_{\mathrm{J}}(s) \cdot \mathcal{H}_{\mathrm{J}}(s) .
\end{aligned}
$$

Furthermore, the convolution of the inverse transform is

$$
T\left\{T^{-1}\{f\} * T^{-1}\{h\}\right\}=\frac{1}{\varphi(s)} f \cdot h .
$$


Table 1 Integral transform of some basic functions

\begin{tabular}{ll}
\hline$f(t)$ & $T\{f(t), s\}=\mathcal{F}_{\mathbf{J}}(s)$ \\
\hline$c$ & $\frac{\varphi(s)}{\psi(s)} c, c \in \mathbb{R}$ \\
$t$ & $\frac{\varphi(s)}{\psi(s)^{2}}$ \\
$t^{\alpha}$ & $\frac{\Gamma(\alpha+1) \varphi(s)}{\psi(s) \alpha+1}, \alpha>0$ \\
$\sin t$ & $\frac{\varphi(s)}{\psi(s)^{2}+1}$ \\
$\cos t$ & $\frac{\varphi(s) \psi(s)}{\psi(s)^{2}+1}$ \\
\hline
\end{tabular}

Hence,

$$
T^{-1}\{f \cdot h\}=\varphi(s) T^{-1}\{f\} * T^{-1}\{h\} .
$$

Table 1 shows the general integral transforms of some basic functions.

The two parameter Mittag-Leffler function is stated as [3]

$$
E_{\eta, \sigma}(t)=\sum_{k=0}^{\infty} \frac{z^{k}}{\Gamma(\eta k+\sigma)}, \quad z \in \mathbb{C}, \operatorname{Re}(\eta)>0 \text { and } \operatorname{Re}(\sigma)>0
$$

A variant of the Mittag-Leffler function is given by Prabhakar as [36]

$$
E_{\eta, \sigma}^{\gamma}(z)=\sum_{k=0}^{\infty} \frac{\gamma_{k}}{\Gamma(\eta k+\sigma)} \frac{z^{k}}{k !}, \quad z \in \mathbb{C}, \operatorname{Re}(\eta)>0, \operatorname{Re}(\sigma)>0 \text { and } \operatorname{Re}(\gamma)>0,
$$

$\gamma_{k}$ is the Pochhammer symbol.

Definition $2([19,22])$ The Atangana-Baleanu fractional derivative is defined by

$$
{ }_{a}^{A B C} D_{t}^{\eta}(f(t))=\frac{\mathcal{K}(\eta)}{1-\eta} \int_{a}^{t} f^{\prime}(x) E_{\eta}\left(\frac{\eta}{\eta-1}(t-x)^{\eta}\right) d x
$$

another version of the Atangana-Baleanu derivative is stated as

$$
{ }_{a}^{A B R} D_{t}^{\eta}(f(t))=\frac{\mathcal{K}(\eta)}{1-\eta} \frac{d}{d t} \int_{a}^{t} f(x) E_{\eta}\left(\frac{\eta}{\eta-1}(t-x)^{\eta}\right) d x
$$

where $\eta \in(0,1)$ and $\mathcal{K}(\eta)$ represents the normalization function with the property $\mathcal{K}(0)=$ $\mathcal{K}(1)=1$.

Definition 3 ([19, 22]) The fractional integral associate to the fractional derivative of Atangana-Baleanu is defined by

$$
{ }_{a}^{A B} I_{t}^{\eta}(f(t))=\frac{1-\eta}{\mathcal{K}(\eta)} f(t)+\frac{\eta}{\mathcal{K}(\eta) \Gamma(\eta)} \int_{a}^{t} f(x)(t-x)^{\eta-1} d x
$$

When $\eta=0$ we recover the initial function, and if $\eta=1$, we obtain the ordinary integral. 


\section{New general transform for Atangana-Baleanu fractional derivatives}

We present the main results of our research in this section.

Lemma 1 Let $0<\eta<1$ and $\lambda \in \mathbb{R}$ such that $\psi(s)<|\lambda|^{\frac{1}{\eta}}$, then

$$
T\left\{t^{\sigma-1} E_{\eta, \sigma}^{\gamma}\left(\lambda t^{\eta}\right), s\right\}=\frac{\varphi(s)}{\psi(s)^{\sigma}} \frac{1}{\left(1-\frac{\lambda}{\psi(s)^{\eta}}\right)^{\gamma}}, \quad \psi(s)>0
$$

Proof The new general integral transform of the function $t^{\sigma-1} E_{\eta, \sigma}^{\gamma}\left(\lambda t^{\eta}\right)$ yields

$$
\begin{aligned}
T\left\{t^{\sigma-1} E_{\eta, \sigma}^{\gamma}\left(\lambda t^{\eta}\right), s\right\} & =\varphi(s) \int_{0}^{\infty} t^{\sigma-1} E_{\eta, \sigma}^{\gamma}\left(\lambda t^{\eta}\right) e^{-\psi(s) t} d t \\
& =\varphi(s) \int_{0}^{\infty} t^{\sigma-1} \sum_{k=0}^{\infty} \frac{\gamma_{k}}{\Gamma(\eta k+\sigma)} \frac{\left(\lambda t^{\eta}\right)^{k}}{k !} e^{-\psi(s) t} d t \\
& =\sum_{k=0}^{\infty} \frac{\gamma_{k}}{\Gamma(\eta k+\sigma)} \frac{\lambda^{k}}{k !} \varphi(s) \int_{0}^{\infty} t^{\eta k+\sigma-1} e^{-\psi(s) t} d t \\
& =\sum_{k=0}^{\infty} \frac{\gamma_{k}}{\Gamma(\eta k+\sigma)} \frac{\lambda^{k}}{k !} T\left\{t^{\eta k+\sigma-1}\right\},
\end{aligned}
$$

thus,

$$
\begin{aligned}
T\left\{t^{\sigma-1} E_{\eta, \sigma}^{\gamma}\left(\lambda t^{\eta}\right), s\right\} & =\sum_{k=0}^{\infty} \frac{\gamma_{k}}{\Gamma(\eta k+\sigma)} \frac{\lambda^{k}}{k !} T\left\{t^{\eta k+\sigma-1}\right\} \\
& =\sum_{k=0}^{\infty} \frac{\gamma_{k}}{\Gamma(\eta k+\sigma)} \frac{\lambda^{k}}{k !} \frac{\Gamma(\eta k+\sigma) \varphi(s)}{\psi(s)^{\eta k+\sigma}} \\
& =\frac{\varphi(s)}{\psi(s)^{\sigma}} \sum_{k=0}^{\infty} \frac{\gamma_{k}}{k !}\left(\frac{\lambda}{\psi(s)^{\eta}}\right)^{k},
\end{aligned}
$$

since $q(s)<|\lambda|^{\frac{1}{\eta}}$, it follows that

$$
T\left\{t^{\sigma-1} E_{\eta, \sigma}^{\gamma}\left(\lambda t^{\eta}\right), s\right\}=\frac{\varphi(s)}{\psi(s)^{\sigma}} \frac{1}{\left(1-\frac{\lambda}{\psi(s)^{\eta}}\right)^{\gamma}} .
$$

Corollary 1 Under the same conditions of Lemma (1), we have the new general transform of the function $E_{\eta}\left(\lambda t^{\eta}\right)$ as

$$
T\left\{E_{\eta}\left(\lambda t^{\eta}\right)\right\}=\frac{\varphi(s)}{\psi(s)} \frac{\psi(s)^{\eta}}{\psi(s)^{\eta}-\lambda},
$$

and the new general transform of the function $t^{\sigma-1} E_{\eta}\left(\lambda t^{\eta}\right)$ as

$$
T\left\{t^{\sigma-1} E_{\eta}\left(\lambda t^{\eta}\right)\right\}=\frac{\varphi(s) \psi(s)^{\eta-\sigma}}{\psi(s)^{\eta}-\lambda} .
$$


Proof We have

$$
T\left\{E_{\eta}\left(\lambda t^{\eta}\right)\right\}=T\left\{E_{\eta, 1}^{1}\left(\lambda t^{\eta}\right), s\right\}=\frac{\varphi(s)}{\psi(s)} \frac{1}{1-\frac{\lambda}{\psi(s)^{\eta}}}
$$

and

$$
T\left\{t^{\sigma-1} E_{\eta}\left(\lambda t^{\eta}\right)\right\}=T\left\{t^{\sigma-1} E_{\eta, \sigma}^{1}\left(\lambda t^{\eta}\right), s\right\}=\frac{\varphi(s) \psi(s)^{\eta-\sigma}}{\psi(s)^{\eta}-\lambda} .
$$

Remark 1 Lemma (1) generalizes the results obtained in [37] for the Sumudu transform and in [38] for the Shehu transform of the function $t^{\sigma-1} E_{\eta, \sigma}^{\gamma}\left(\lambda t^{\eta}\right)$. Indeed, when $\varphi(s)=\frac{1}{s}$ and $\psi(s)=\frac{1}{s}$ the Sumudu transform is

$$
S\left\{t^{\sigma-1} E_{\eta, \sigma}^{\gamma}\left(\lambda t^{\eta}\right)\right\}=\frac{\frac{1}{s}}{\left(\frac{1}{s}\right)^{\sigma}} \frac{1}{\left(1-\frac{\lambda}{\left(\frac{1}{s}\right)^{\eta}}\right)^{\gamma}}=s^{\sigma-1}\left(1-\lambda s^{\eta}\right)^{-\gamma},
$$

for $\varphi(s)=1$ and $\psi(s)=\frac{s}{u}$, we obtain the Shehu transform,

$$
S H\left\{t^{\sigma-1} E_{\eta, \sigma}^{\gamma}\left(\lambda t^{\eta}\right)\right\}=\frac{1}{\left(\frac{s}{u}\right)^{\sigma}} \frac{1}{\left(1-\frac{\lambda}{\left(\frac{s}{u}\right)^{\eta}}\right)^{\gamma}}=\left(\frac{u}{s}\right)^{\sigma}\left(1-\lambda\left(\frac{u}{s}\right)^{\eta}\right)^{-\gamma} .
$$

Obviously, when $\gamma=1$, we get the Sumudu and Shehu transform of the function $t^{\sigma-1} E_{\eta, \sigma}\left(\lambda t^{\eta}\right)$.

Henceforth, we suppose that the function $f(t) \in A \cap H^{1}(a, b)$ such that $H^{1}(a, b)$ is a Sobolev space of order one defined by

$$
H^{1}(a, b)=\left\{f \in L^{2}(a, b): f^{\prime} \in L^{2}(a, b)\right\} .
$$

Theorem 3 The new general integral transform of the Atangana-Baleanu derivative stated in (3) is given as

$$
T\left\{{ }_{0}^{A B C} D_{t}^{\eta}(f(t))\right\}=\frac{\mathcal{K}(\eta)}{1-\eta} \frac{\psi(s)^{\eta}}{\psi(s)^{\eta}+\frac{\eta}{1-\eta}}\left[\mathcal{F}_{\mathrm{J}}(s)-\frac{\varphi(s)}{\psi(s)} f(0)\right] .
$$

Proof Let $\mathcal{F}_{\mathrm{J}}(s)$ be the new general transform of the function $f(t)$. Let us observe that in definition (3) we have a convolution integral,

$$
\int_{0}^{t} f^{\prime}(x) E_{\eta}\left(\frac{\eta}{\eta-1}(t-x)^{\eta}\right) d x=f^{\prime}(t) * E_{\eta}\left(\frac{\eta}{\eta-1} t^{\eta}\right)
$$

then one has

$$
\begin{aligned}
T\left\{{ }_{0}^{A B C} D_{t}^{\eta}(f(t))\right\} & =T\left\{\frac{\mathcal{K}(\eta)}{1-\eta} \int_{0}^{t} f^{\prime}(x) E_{\eta}\left(\frac{\eta}{\eta-1}(t-x)^{\eta}\right) d x\right\} \\
& =\frac{\mathcal{K}(\eta)}{1-\eta} T\left\{f^{\prime}(t) * E_{\eta}\left(\frac{\eta}{\eta-1} t^{\eta}\right)\right\}
\end{aligned}
$$




$$
=\frac{\mathcal{K}(\eta)}{1-\eta} \frac{1}{\varphi(s)} T\left\{f^{\prime}(t)\right\} \cdot T\left\{E_{\eta}\left(\frac{\eta}{\eta-1} t^{\eta}\right)\right\} .
$$

Using Theorem 1 and applying the result obtained in Corollary 1 , then

$$
\begin{aligned}
T\left\{{ }_{0}^{A B C} D_{t}^{\eta}(f(t))\right\} & =\frac{\mathcal{K}(\eta)}{1-\eta} \frac{1}{\varphi(s)}\left[\psi(s) \mathcal{F}_{\mathrm{J}}(s)-\varphi(s) f(0)\right] \frac{\varphi(s)}{\psi(s)} \frac{\psi(s)^{\eta}}{\psi(s)^{\eta}+\frac{\eta}{1-\eta}} \\
& =\frac{\mathcal{K}(\eta)}{1-\eta} \frac{\psi(s)^{\eta}}{\psi(s)^{\eta}+\frac{\eta}{1-\eta}}\left[\mathcal{F}_{\mathrm{J}}(s)-\frac{\varphi(s)}{\psi(s)} f(0)\right] .
\end{aligned}
$$

\section{Corollary 2}

- If $\varphi(s)=1$ and $\psi(s)=s$, then this new transform gives the Laplace transform, see $[19,20]$,

$$
\begin{aligned}
\left.L_{0}^{A B C} D_{t}^{\eta}(f(t))\right\} & =\frac{\mathcal{K}(\eta)}{1-\eta} \times \frac{s^{\eta}}{s^{\eta}+\frac{\eta}{1-\eta}}\left[\mathcal{F}_{\mathrm{J}}(s)-\frac{1}{s} f(0)\right] \\
& =\frac{\mathcal{K}(\eta)}{1-\eta} \frac{s^{\eta} \mathcal{F}_{\mathrm{J}}(s)-s^{\eta-1} f(0)}{s^{\eta}+\frac{\eta}{1-\eta}} .
\end{aligned}
$$

- If $\varphi(s)=s$ and $\psi(s)=\frac{1}{s}$, we have the Elzaki transform [39],

$$
\begin{aligned}
E\left\{{ }_{0}^{A B R} D_{t}^{\eta}(u(t))\right\} & =\frac{\mathcal{K}(\eta)}{1-\eta} \frac{\left(\frac{1}{s}\right)^{\eta}}{\left(\frac{1}{s}\right)^{\eta}+\frac{\eta}{1-\eta}}\left[\mathcal{F}_{\mathrm{J}}(s)-\frac{s}{\frac{1}{s}} f(0)\right] \\
& =\frac{\mathcal{K}(\eta)}{1-\eta} \frac{1}{1+\frac{\eta}{1-\eta} s^{\eta}}\left[\mathcal{F}_{\mathrm{J}}(s)-s^{2} f(0)\right] .
\end{aligned}
$$

- If $\varphi(s)=\psi(s)=\frac{1}{s}$, the new transform coincides with the Sumudu transform [40],

$$
\begin{aligned}
\left.S_{\{0}^{A B C} D_{t}^{\eta}(u(t))\right\} & =\frac{\mathcal{K}(\eta)}{1-\eta} \frac{\frac{1}{s^{\eta}}}{\frac{1}{s^{\eta}}+\frac{\eta}{1-\eta}}\left[\mathcal{F}_{\mathrm{J}}(s)-f(0)\right] \\
& =\frac{\mathcal{K}(\eta)}{1-\eta} \frac{1}{1+\frac{\eta}{1-\eta} s^{\eta}}\left[\mathcal{F}_{\mathrm{J}}(s)-f(0)\right]
\end{aligned}
$$

- If $\varphi(s)=1$ and $\psi(s)=\frac{s}{u}$, then the Shehu transform of the Atangana-Baleanu fractional derivative in Caputo sense [40] is obtained,

$$
\begin{aligned}
S H\left\{{ }_{0}^{A B C} D_{t}^{\eta}(u(t))\right\} & =\frac{\mathcal{K}(\eta)}{1-\eta} \frac{\left(\frac{s}{u}\right)^{\eta}}{\left(\frac{s}{u}\right)^{\eta}+\frac{\eta}{1-\eta}}\left[\mathcal{F}_{\mathrm{J}}(s)-\frac{1}{\frac{s}{u}} f(0)\right] \\
& =\frac{\mathcal{K}(\eta)}{1-\eta} \frac{\left(\frac{s}{u}\right)^{\eta} \mathcal{F}_{\mathrm{J}}(s)-\left(\frac{s}{u}\right)^{\eta-1} f(0)}{\left(\frac{s}{u}\right)^{\eta}+\frac{\eta}{1-\eta}} .
\end{aligned}
$$

Theorem 4 The new general integral transform of the Atangana-Baleanu derivative stated in (4) is given as

$$
\left.T_{0}^{A B R} D_{t}^{\eta}(u(t))\right\}=\frac{\mathcal{K}(\eta)}{1-\eta} \frac{\psi(s)^{\eta}}{\psi(s)^{\eta}+\frac{\eta}{1-\eta}} \mathcal{F}_{\mathrm{J}}(s)
$$


Proof Let $\mathcal{F}_{\mathrm{J}}(s)$ be the generalized transform of the function $f(t)$. We have

$$
\begin{aligned}
T\left\{_{0}^{A B R} D_{t}^{\eta}(f(t))\right\} & =T\left\{\frac{\mathcal{K}(\eta)}{1-\eta} \frac{d}{d t} \int_{a}^{t} f(x) E_{\eta}\left(\frac{\eta}{\eta-1}(t-x)^{\eta}\right) d x\right\} \\
& =\frac{\mathcal{K}(\eta)}{1-\eta} T\left\{\frac{d}{d t}\left[f(t) * E_{\eta}\left(\frac{\eta}{\eta-1} t^{\eta}\right)\right]\right\} \\
& =\frac{\mathcal{K}(\eta)}{1-\eta}\left[\psi(s) T\left\{f(t) * E_{\eta}\left(\frac{\eta}{\eta-1} t^{\eta}\right)\right\}-\varphi(s) T\left\{f(0) * E_{\eta}(0)\right\}\right],
\end{aligned}
$$

hence,

$$
T\left\{{ }_{0}^{A B R} D_{t}^{\eta}(f(t))\right\}=\frac{\mathcal{K}(\eta)}{1-\eta} \frac{\psi(s)^{\eta}}{\psi(s)^{\eta}+\frac{\eta}{1-\eta}} \mathcal{F}_{\mathrm{J}}(s) .
$$

\section{Corollary 3}

- If $\varphi(s)=1$ and $\psi(s)=s$, then the Laplace transform is given by $[19,20]$

$$
L\left\{{ }_{0}^{A B R} D_{t}^{\eta}(f(t))\right\}=\frac{\mathcal{K}(\eta)}{1-\eta} \frac{s^{\eta}}{s^{\eta}+\frac{\eta}{1-\eta}} \mathcal{F}_{\mathrm{J}}(s) .
$$

- If $\varphi(s)=s$ and $\psi(s)=\frac{1}{s}$, we have the Elzaki transform [39],

$$
\left.E_{0}^{A B C} D_{t}^{\eta}(f(t))\right\}=\frac{\mathcal{K}(\eta)}{1-\eta} \frac{1}{1+\frac{\eta}{1-\eta} s^{\eta}} \mathcal{F}_{\mathrm{J}}(s)
$$

- If $\varphi(s)=\psi(s)=\frac{1}{s}$, we get the Sumudu transform as follows [40]:

$$
S\left\{\begin{array}{c}
A B C \\
0
\end{array} D_{t}^{\eta}(f(t))\right\}=\frac{\mathcal{K}(\eta)}{1-\eta} \frac{\left(\frac{1}{s}\right)^{\eta}}{\left(\frac{1}{s}\right)^{\eta}+\frac{\eta}{1-\eta}} \mathcal{F}_{\mathrm{J}}(s) .
$$

- If $\varphi(s)=1$ and $\psi(s)=\frac{s}{u}$, then the Shehu transform of the Atangana-Baleanu fractional derivative in Riemann-Liouville sense [40] is given as

$$
S H\left\{{ }_{0}^{A B C} D_{t}^{\eta}(f(t))\right\}=\frac{\mathcal{K}(\eta)}{1-\eta} \frac{\left(\frac{s}{u}\right)^{\eta}}{\left(\frac{s}{u}\right)^{\eta}+\frac{\eta}{1-\eta}} \mathcal{F}_{\mathrm{J}}(s)
$$

Lemma 2 The new general integral transform of the function $t^{\eta-1}$ is given as

$$
T\left\{t^{\eta-1}\right\}=\frac{\Gamma(\eta) \varphi(s)}{\psi(s)^{\eta}}, \quad \eta>0
$$

Proof Applying the new general integral transform of the function $t^{\eta-1}$, we get

$$
T\left\{t^{\eta-1}\right\}=\varphi(s) \int_{0}^{\infty} t^{\eta-1} e^{-\psi(s) t} d t .
$$

Let $\Gamma(\eta)$ be the Gamma function defined by

$$
\Gamma(\eta)=\int_{0}^{\infty} u^{\eta-1} e^{-u} d u
$$


Setting $u=\psi(s) t$ implies $d u=\psi(s) d t$, then

$$
\Gamma(\eta)=\int_{0}^{\infty}(\psi(s) t)^{\eta-1} e^{-\psi(s) t} \psi(s) d t=\psi(s)^{\eta} \int_{0}^{\infty} t^{\eta-1} e^{-\psi(s) t} d t,
$$

thus,

$$
\int_{0}^{\infty} t^{\eta-1} e^{-\psi(s) t} d t=\frac{\Gamma(\eta)}{\psi(s)^{\eta}} .
$$

Hence,

$$
T\left\{t^{\eta-1}\right\}=\frac{\Gamma(\eta) \varphi(s)}{\psi(s)^{\eta}} .
$$

Remark 2 Through the result in Lemma (2), we can deduce the Sumudu transform of the function $t^{\eta-1}$ (see [41]) and the Shehu transform (see [40]).

Theorem 5 The general transform of the Atangana-Baleanu fractional integral of the function $f(t)$ is given as

$$
T\left\{{ }_{0}^{A B} I_{t}^{\eta}(f(t))\right\}=\frac{1}{\mathcal{K}(\eta)} \frac{(1-\eta) \psi(s)^{\eta}+\eta}{\psi(s)^{\eta}} \mathcal{F}_{\mathrm{J}}(s) .
$$

Proof Let $\mathcal{F}_{\mathrm{J}}(s)$ be the new general transform of the function $f(t)$, we have

$$
\begin{aligned}
T\left\{{ }_{0}^{A B} I_{t}^{\eta}(f(t))\right\} & =\frac{1-\eta}{\mathcal{K}(\eta)} T\{f(t)\}+\frac{\eta}{\mathcal{K}(\eta) \Gamma(\eta)} T\left\{\int_{0}^{t} f(x)(t-x)^{\eta-1} d x\right\} \\
& =\frac{1-\eta}{\mathcal{K}(\eta)} T\{f(t)\}+\frac{\eta}{\mathcal{K}(\eta) \Gamma(\eta)} T\left\{f(t) * t^{\eta-1}\right\} .
\end{aligned}
$$

According to the convolution theorem (2) and Lemma (2), we obtain,

$$
\begin{aligned}
T\left\{{ }_{0}^{A B} I_{t}^{\eta}(f(t))\right\} & =\frac{1-\eta}{\mathcal{K}(\eta)} \mathcal{F}_{\mathrm{J}}(s)+\frac{\eta}{\mathcal{K}(\eta) \Gamma(\eta)} \frac{1}{\varphi(s)} T\{f(t)\} T\left\{t^{\eta-1}\right\} \\
& =\frac{1-\eta}{\mathcal{K}(\eta)} \mathcal{F}_{\mathrm{J}}(s)+\frac{\eta}{\mathcal{K}(\eta) \Gamma(\eta)} \frac{1}{\varphi(s)} \mathcal{F}_{\mathrm{J}}(s) \frac{\Gamma(\eta) \varphi(s)}{\psi(s)^{\eta}}
\end{aligned}
$$

then

$$
T\left\{{ }_{0}^{A B} I_{t}^{\eta}(f(t))\right\}=\frac{1}{\mathcal{K}(\eta)} \frac{(1-\eta) \psi(s)^{\eta}+\eta}{\psi(s)^{\eta}} \mathcal{F}_{\mathrm{J}}(s) .
$$

\section{Applications}

We now substantiate the theoretical aspects that we developed in the previous section by providing practical examples.

Example 1 Consider the following fractional initial value problem:

$$
{ }_{0}^{A B C} D_{t}^{\eta}(u(t))=f(t), \quad u(0)=c, c \in \mathbb{R} .
$$


Applying the integral transform on both sides of Eq. (7),

$$
T\left\{{ }_{0}^{A B C} D_{t}^{\eta}(u(t))\right\}=T\{f(t)\},
$$

gives

$$
\frac{\mathcal{K}(\eta)}{1-\eta} \frac{\psi(s)^{\eta}}{\psi(s)^{\eta}+\frac{\eta}{1-\eta}}\left[\mathcal{U}_{\mathrm{J}}(s)-\frac{\varphi(s)}{\psi(s)} u(0)\right]=\mathcal{F}_{\mathrm{J}}(s) .
$$

Therefore,

$$
\mathcal{U}_{\mathrm{J}}(s)=\frac{1-\eta}{\mathcal{K}(\eta)} \frac{\psi(s)^{\eta}+\frac{\eta}{1-\eta}}{\psi(s)^{\eta}} \mathcal{F}_{\mathrm{J}}(s)+\frac{\varphi(s)}{\psi(s)} u(0),
$$

taking the inverse general transform, we obtain

$$
u(t)=T^{-1}\left\{\frac{1-\eta}{\mathcal{K}(\eta)} \frac{\psi(s)^{\eta}+\frac{\eta}{1-\eta}}{\psi(s)^{\eta}} \mathcal{F}_{\mathrm{J}}(s)+\frac{\varphi(s)}{\psi(s)} u(0)\right\}
$$

If $f(t)=t$, the equivalence of (7) is

$$
{ }_{0}^{A B C} D_{t}^{\eta}(u(t))=t, \quad u(0)=c, c \in \mathbb{R},
$$

whose solution is

$$
\begin{aligned}
u(t) & =T^{-1}\left\{\frac{1-\eta}{\mathcal{K}(\eta)}\left(1+\frac{\eta}{1-\eta} \frac{1}{\psi(s)^{\eta}}\right) \frac{\varphi(s)}{\psi(s)}+\frac{\varphi(s)}{\psi(s)} u(0)\right\} \\
& =\frac{1-\eta}{\mathcal{K}(\eta)} T^{-1}\left\{\frac{\varphi(s)}{\psi(s)}+\frac{\eta}{1-\eta} \frac{\varphi(s)}{\psi(s)^{\eta+1}}+\frac{\varphi(s)}{\psi(s)} u(0)\right\} .
\end{aligned}
$$

The above equation reduces to

$$
u(t)=\frac{1-\eta}{\mathcal{K}(\eta)}\left(1+c+\frac{\eta}{1-\eta} \frac{1}{\Gamma(\eta+1)} t^{\eta}\right) .
$$

Example 2 Consider the following nonlinear fractional differential equation:

$$
{ }_{0}^{A B C} D_{t}^{\eta}(u(t))+u(t)=f(t) .
$$

By employing the new general transform in Eq. (9), we get

$$
T\left\{{ }_{0}^{A B C} D_{t}^{\eta}(u(t))\right\}+T\{u(t)\}=T\{f(t)\},
$$

thus,

$$
\frac{\mathcal{K}(\eta)}{1-\eta} \frac{\psi(s)^{\eta}}{\psi(s)^{\eta}+\frac{\eta}{1-\eta}}\left[\mathcal{U}_{\mathrm{J}}(s)-\frac{\varphi(s)}{\psi(s)} u(0)\right]+\mathcal{U}_{\mathrm{J}}(s)=\mathcal{F}_{\mathrm{J}}(s) .
$$


Therefore,

$$
\left(\frac{\mathcal{K}(\eta)}{1-\eta} \frac{\psi(s)^{\eta}}{\psi(s)^{\eta}+\frac{\eta}{1-\eta}}+1\right) \mathcal{U}_{\mathrm{J}}(s)=\frac{\mathcal{K}(\eta)}{1-\eta} \frac{\psi(s)^{\eta}}{\psi(s)^{\eta}+\frac{\eta}{1-\eta}} \frac{\varphi(s)}{\psi(s)} u(0)+\mathcal{F}_{\mathrm{J}}(s)
$$

it follows that

$$
\begin{aligned}
\mathcal{U}_{\mathrm{J}}(s)= & \frac{\mathcal{K}(\eta)}{\mathcal{K}(\eta)+1-\eta} \frac{\psi(s)^{\eta}}{\psi(s)^{\eta}+\frac{\eta}{\mathcal{K}(\eta)+1-\eta}} \frac{\varphi(s)}{\psi(s)} u(0)+\frac{1-\eta}{\mathcal{K}(\eta)+1-\eta} \frac{\psi(s)^{\eta}+\eta}{\psi(s)^{\eta}+\frac{\eta}{\mathcal{K}(\eta)+1-\eta}} \mathcal{F}_{\mathrm{J}}(s), \\
\mathcal{U}_{\mathrm{J}}(s)= & \frac{\mathcal{K}(\eta)}{\mathcal{K}(\eta)+1-\eta} \frac{\psi(s)^{\eta}}{\psi(s)^{\eta}+\frac{\eta}{\mathcal{K}(\eta)+1-\eta}} \frac{\varphi(s)}{\psi(s)} u(0)+\frac{1-\eta}{\mathcal{K}(\eta)+1-\eta} \mathcal{F}_{\mathrm{J}}(s) \\
& +\frac{\eta}{(\mathcal{K}(\eta)+1-\eta)^{2}} \frac{1}{\psi(s)^{\eta}+\frac{\eta}{\mathcal{K}(\eta)+1-\eta}} \mathcal{F}_{\mathrm{J}}(s) .
\end{aligned}
$$

Using the inverse transform, the convolution theorem (2) and Corollary 1, we get

$$
T^{-1}\left\{\frac{\psi(s)^{\eta}}{\psi(s)^{\eta}+\frac{\eta}{\mathcal{K}(\eta)+1-\eta}} \frac{\varphi(s)}{\psi(s)}\right\}=E_{\eta}\left(-\frac{\eta}{\mathcal{K}(\eta)+1-\eta} t^{\eta}\right)
$$

also,

$$
\begin{aligned}
T^{-1}\left\{\frac{1}{\psi(s)^{\eta}+\frac{\eta}{\mathcal{K}(\eta)+1-\eta}} \mathcal{F}_{\mathrm{J}}(s)\right\} & =T^{-1}\left\{\frac{\varphi(s)}{\psi(s)^{\eta}+\frac{\eta}{\mathcal{K}(\eta)+1-\eta}}\right\} * f(t) \\
& =t^{\eta-1} E_{\eta}\left(-\frac{\eta}{\mathcal{K}(\eta)+1-\eta} t^{\eta}\right) * f(t) .
\end{aligned}
$$

Then

$$
\begin{aligned}
u(t)= & T^{-1}\left\{\mathcal{U}_{\mathrm{J}}(s)\right\} \\
= & \frac{\mathcal{K}(\eta)}{\mathcal{K}(\eta)+1-\eta} E_{\eta}\left(-\frac{\eta}{\mathcal{K}(\eta)+1-\eta} t^{\eta}\right) u(0)+\frac{1-\eta}{\mathcal{K}(\eta)+1-\eta} f(t) \\
& +\frac{\eta}{(\mathcal{K}(\eta)+1-\eta)^{2}} t^{\eta-1} E_{\eta}\left(-\frac{\eta}{\mathcal{K}(\eta)+1-\eta} t^{\eta}\right) * f(t) .
\end{aligned}
$$

Note that, when $\eta \rightarrow 1$ and $f(t)=e^{-2 t}$, then the exact solution is

$$
u(t)=(1+u(0)) e^{-t}-e^{-2 t} .
$$

Example 3 We now consider Eq. (9) with the Riemann-Liouville derivative,

$$
{ }_{0}^{\mathrm{ABR}} D_{t}^{\eta}(u(t))+u(t)=f(t) .
$$

Applying the new general transform in Eq. (10) yields

$$
\left(\frac{\mathcal{K}(\eta)}{1-\eta} \frac{\psi(s)^{\eta}}{\psi(s)^{\eta}+\frac{\eta}{1-\eta}}+1\right) \mathcal{U}_{\mathrm{J}}(s)=T\{f(t)\}
$$


Therefore,

$$
\begin{aligned}
& \mathcal{U}_{\mathrm{J}}(s)=\frac{(1-\eta) \psi(s)^{\eta}+\eta}{(\mathcal{K}(\eta)+1-\eta) \psi(s)^{\eta}+\eta} \mathcal{F}_{\mathrm{J}}(s) \\
& u(t)=T^{-1}\left\{\frac{(1-\eta) \psi(s)^{\eta}+\eta}{(\mathcal{K}(\eta)+1-\eta) \psi(s)^{\eta}+\eta} \mathcal{F}_{\mathrm{J}}(s)\right\}
\end{aligned}
$$

If $f(t)=\sin (t),(10)$ becomes

$$
{ }_{0}^{A B R} D_{t}^{\eta}(u(t))+u(t)=\sin (t), \quad 0<\eta \leq 1,
$$

whose exact solution is

$$
u(t)=T^{-1}\left\{\frac{(1-\eta) \psi(s)^{\eta}+\eta}{(\mathcal{K}(\eta)+1-\eta) \psi(s)^{\eta}+\eta} \frac{\varphi(s)}{\psi(s)^{2}+1}\right\}
$$

In particular, when $\eta \rightarrow 1$, we obtain

$$
\begin{aligned}
u(t) & =T^{-1}\left\{\frac{1}{\psi(s)+1} \frac{\varphi(s)}{\psi(s)^{2}+1}\right\} \\
& =T^{-1}\left\{0.5 \frac{\varphi(s)}{\psi(s)+1}-0.5 \frac{\psi(s) \varphi(s)}{\psi(s)^{2}+1}+0.5 \frac{\varphi(s)}{\psi(s)^{2}+1}\right\},
\end{aligned}
$$

with the exact solution

$$
u(t)=0.5\left(e^{-t}-\cos t+\sin t\right) .
$$

\section{Conclusion}

We explored the feasibility of applying the generalized integral transform (Jafari transform) in fractional calculus, the Atangana-Baleanu derivative with its corresponding integral is used as a case in point. It is proved that imposing certain conditions on the Jafari transform leads to other integral transforms. To prove the applicability of this generalised integral transform, practical examples are given. This generalised integral transform resembles other integral transforms in that it is easy to implement and offers the convenience of using a table in the solution procedure of differential equations.

Acknowledgements

We would like thank the editor and reviewers for their thoughtful and detailed comments on our paper.

Funding

Not applicable.

Availability of data and materials

Not applicable.

Competing interests

The authors declare that they have no competing interests.

Authors' contributions

All authors have read and approved the final manuscript.

\section{Author details}

'Department of Mathematics, University of Hassiba Benbouali, Chlef, Algeria. ${ }^{2}$ Department of Mathematics, University of Mazandaran, Babolsar, Iran. ${ }^{3}$ Department of Mathematical Sciences, University of South Africa, UNISA0003, Pretoria, South Africa. ${ }^{4}$ Department of Medical Research, China Medical University Hospital, China Medical University, Taichung, 110122, Taiwan. 


\section{Publisher's Note}

Springer Nature remains neutral with regard to jurisdictional claims in published maps and institutional affiliations.

\section{Received: 31 May 2021 Accepted: 3 August 2021 Published online: 19 August 2021}

\section{References}

1. Samko, S.G., Kilbas, A.A., Marichev, O.I.: Fractional Integrals and Derivatives, Theory and Applications. Gordon \& Breach, Yverdon (1993)

2. Miller, K.S., Ross, B.: An Introduction to the Fractional Calculus and Fractional Differential Equations. Wiley, New York (1993)

3. Podlubny, I.: Fractional Differential Equations: An Introduction to Fractional Derivatives, Fractional Differential Equations, to Methods of Their Solution and Some of Their Applications. Academic Press, San Diego (1998) 198, 10

4. Karapinar, E., Binh, H.D., Luc, N.H., Can, N.H.: On continuity of the fractional derivative of the time-fractional semilinear pseudo-parabolic systems. Adv. Differ. Equ. 2021, 70 (2021)

5. Adiguzel, R.S., Aksoy, U., Karapınar, E., Erhan, I.M.: On the solution of a boundary value problem associated with a fractional differential equation. Math. Methods Appl. Sci. (2020). https://doi.org/10.1002/mma.6652

6. Afshari, H., Karapınar, E.: A discussion on the existence of positive solutions of the boundary value problems via $\psi$-Hilfer fractional derivative on b-metric spaces. Adv. Differ. Equ. 2020, Article number 616 (2020)

7. Afshari, H., Kalantari, S., Karapinar, E.: Solution of fractional differential equations via coupled fixed point. Electron. J. Differ. Equ. 2015, No. 286, pp. 1-12 (2015)

8. Alqahtani, B., Aydi, H., Karapınar, E., Rakocevic, V:: A solution for Volterra fractional integral equations by hybrid contractions. Mathematics 7, 694 (2019)

9. Abdeljawad, A., Agarwal, R.P., Karapinar, E., Kumari, P.S.: Solutions of the nonlinear integral equation and fractional differential equation using the technique of a fixed point with a numerical experiment in extended b-metric space. Symmetry 11, 686 (2019)

10. Afshari, H., Atapour, M., Karapinar, E.: A discussion on a generalized Geraghty multi-valued mappings and applications. Adv. Differ. Equ. 2020, 356 (2020)

11. Hosseini, V.R., Koushki, M., Zou, W.N.: The meshless approach for solving 2D variable-order time-fractional advection-diffusion equation arising in anomalous transport. Eng. Comput. (2021). https://doi.org/10.1007/s00366-021-01379-7

12. Hosseini, V.R., Yousefi, F., Zou, W.N.: The numerical solution of high dimensional variable-order time fractional diffusion equation via the singular boundary method. J. Adv. Res. (2020). https://doi.org/10.1016/j.jare.2020.12.0

13. Hosseini, V.R., Shivanian, E., Chen, W.: Local integration of 2-D fractional telegraph equation via local radial point interpolant approximation. Eur. Phys. J. Plus 130(2), 1-21 (2015)

14. Tuan, N.H., Mohammadi, H., Rezapour, Sh.: A mathematical model for COVID-19 transmission by using the Caputo fractional derivative. Chaos Solitons Fractals 140, 110107 (2020)

15. Kilbas, A.A.: Hadamard-type fractional calculus. J. Korean Math. Soc. 38(6), 1191-1204 (2001)

16. Karapinar, E., Moustafa, S.I., Shehata, A., Agarwal, R.P.: Fractional hybrid differential equations and coupled fixed-point results for $\alpha$-admissible $F\left(\psi_{1}, \psi_{2}\right)$-contractions in M-metric spaces. Discrete Dyn. Nat. Soc. 2020, Article ID 7126045 (2020)

17. Caputo, M., Fabrizio, M.: A new definition of fractional derivative without singular kernel. Prog. Fract. Differ. Appl. 1, 73-85 (2015)

18. Losada, J., Nieto, J.J.: Properties of a new fractional derivative without singular kernel. Prog. Fract. Differ. Appl. 1, 87-92 (2015)

19. Atangana, A., Baleanu, D.: New fractional derivatives with nonlocal and non-singular kernel: theory and application to heat transfer model. Therm. Sci. 20, 763-769 (2016)

20. Atangana, A., Koca, l.: Chaos in a simple nonlinear system with Atangana-Baleanu derivatives with fractional order. Chaos Solitons Fractals 89, 447-454 (2016)

21. Boudaoui, A., El hadj Moussa, Y., Hammouch, Z., Ullah, S.: A fractional-order model describing the dynamics of the novel coronavirus (COVID-19) with nonsingular kernel. Chaos Solitons Fractals 146, 110859 (2021)

22. Atangana, A.: On the new fractional derivative and application to nonlinear Fisher's reaction diffusion equation. Appl. Math. Comput. 273, 948-956 (2016)

23. Al-Refai, M.: On weighted Atangana-Baleanu fractional operators. Adv. Differ. Equ. 2020, 3 (2020)

24. Lazreg, J.E., Abbas, S., Karapinar, E.: Impulsive Caputo-Fabrizio fractional differential equations in b-metric spaces. Mathematics 19, 363-372 (2021)

25. Adiguzel, R.S., Aksoy, U., Karapinar, E., Erhan, I.M.: Uniqueness of solution for higher-order nonlinear fractional differential equations with multi-point and integral boundary conditions. Rev. R. Acad. Cienc. Exactas Fís. Nat., Ser. A Mat. 115, 155 (2021). https://doi.org/10.1007/s13398-021-01095-3

26. Salim, A., Benchohra, M., Karapinar, E., Lazreg, J.E.: Existence and Ulam stability for impulsive generalised Hilfer-type fractional differential equations. Adv. Differ. Equ. 2020, 601 (2020)

27. Derbazi, C., Baitiche, Z., Benchohra, M.: Cauchy problem with $\psi$-Caputo fractional derivative in Banach spaces. Adv. Theory Nonlinear Anal. Appl. 4(4), 349-360 (2021)

28. Baitiche, Z., Derbazi, C., Benchohra, M.: $\psi$-Caputo fractional differential equations with multi-point boundary conditions by topological degree theory. Results Nonlinear Anal. 3(4), 167-178 (2021)

29. Dyke, P.: An Introduction to Laplace Transforms and Fourier Series. Springer, London (2014)

30. Yang, X.-J.: Theory and Applications of Special Functions for Scientists and Engineers. Springer, Singapore (2021)

31. Elzaki, T.M.: The new integral transform 'Elzaki transform'. Glob. J. Pure Appl. Math. 7, 57-64 (2011)

32. Watugala, G.K.: Sumudu transform: a new integral transform to solve differential equations and control engineering problems. Int. J. Math. Educ. Sci. Technol. 24(1), 35-43 (1993)

33. Maitama, S., Zhao, W.: New integral transform: Shehu transform a generalization of Sumudu and Laplace transform for solving differential equations. Int. J. Anal. Appl. 17(2), 167-190 (2019)

34. Manjarekar, S., Bhadane, A.P.: Generalized Elzaki-Tarig transformation and its applications to new fractional derivative with non singular kernel. Prog. Fract. Differ. Appl. 3(3), 227-232 (2017) 
35. Jafari, H.: A new general integral transform for solving integral equations. J. Adv. Res. (2020). https://doi.org/10.1016/j.jare.2020.08.016

36. Prabhakar, T.R.: A singular integral equation with generalized Mittag-Leffler function in the kernel. Yokohama Math. J. 19, 7-15 (1971)

37. Gupta, V.G., Sharma, B.: Application of Sumudu transform in reaction-diffusion systems and nonlinear waves. Appl. Math. Sci. 4(9), 435-446 (2010)

38. Belgacem, R., Baleanu, D., Bokhari, A.: Shehu transform and applications to Caputo fractional differential equations. Int. J. Anal. Appl. 17(6), 917-927 (2019)

39. Yavuz, M., Abdeljawad, T.: Nonlinear regularized long-wave models with a new integral transformation applied to the fractional derivative with power and Mittag-Leffler kernel. Adv. Differ. Equ. 2020, 367 (2020)

40. Bokhari, A., Baleanu, D., Belgacema, R.: Application of Shehu transform to Atangana-Baleanu derivatives. J. Math. Comput. Sci. 20, 101-107 (2020)

41. Belgacem, F.M., Karaballi, A.A., Kalla, S.: Analytical investigations of the Sumudu transform and applications to integral production equations. Math. Probl. Eng. 2003(3), 103-118 (2003)

Submit your manuscript to a SpringerOpen ${ }^{\circ}$ journal and benefit from:

- Convenient online submission

- Rigorous peer review

- Open access: articles freely available online

- High visibility within the field

- Retaining the copyright to your article

Submit your next manuscript at $\boldsymbol{\triangleright}$ springeropen.com 